University of Wollongong

Research Online

Faculty of Engineering and Information

Faculty of Engineering and Information

Sciences - Papers: Part A

Sciences

$1-1-2015$

Effect of pre-quenching process on microstructure and mechanical properties in a Nb-microalloyed low carbon Q-P steel

Jun Zhang

Northeastern University

Hua Ding

Northeastern University

Jingwei Zhao

University of Wollongong, jzhao@uow.edu.au

Follow this and additional works at: https://ro.uow.edu.au/eispapers

Part of the Engineering Commons, and the Science and Technology Studies Commons

Research Online is the open access institutional repository for the University of Wollongong. For further information contact the UOW Library: research-pubs@uow.edu.au 


\title{
Effect of pre-quenching process on microstructure and mechanical properties in a Nb-microalloyed low carbon Q-P steel
}

\author{
Abstract \\ A refined microstructure consisting of martensite and retained austenite at room temperature has been \\ produced in a $\mathrm{Nb}$-microalloyed low carbon Si-Mn steel by a novel heat-treatment, pre-quenching prior to \\ quenching and partitioning processes (Q\&Q-P). The results showed that compared with the conventional \\ quenching and partitioning steel the mechanical properties of steel obtained by the novel treatment have \\ been significantly improved, with a good combination of ultimate tensile strength (1000MPa) and total \\ elongation (above $30 \%$ ). Meanwhile, the volume fraction of retained austenite has been increased. It was \\ found that the improvement of mechanical properties was mainly attributed to the enhanced TRIP effect \\ due to the relatively high fraction of metastable retained austenite at room temperature. The increased \\ stability of austenite results from the $C$ and Mn partitioning during inter-critical annealing, which \\ increased the chemical stability of austenite. The formation of refined austenite at inter-critical annealing \\ also had a positive effect on the stability of the austenite. As a consequence, the volume fraction of \\ retained austenite at room temperature was significantly increased. Compared with the Q-P steel, the \\ Q\&Q-P steel exhibited higher work hardening exponents during the stage of TRIP effect and had the \\ higher ductility.

\section{Keywords} \\ nb, microalloyed, low, carbon, process, quenching, pre, effect, q, p, steel, microstructure, mechanical, \\ properties

\section{Disciplines} \\ Engineering | Science and Technology Studies

\section{Publication Details} \\ Zhang, J., Ding, H. \& Zhao, J. (2015). Effect of pre-quenching process on microstructure and mechanical \\ properties in a Nb-microalloyed low carbon Q-P steel. Materials Science Forum, 816 729-735.
}




\title{
The effect of pre-quenching process on microstructure and mechanical
}

\section{properties in a Nb-microalloyed low carbon Q-P steel}

\author{
Jun Zhang ${ }^{1, \text { a }}$, Hua Ding ${ }^{1, b}$, Jingwei Zhao ${ }^{2, c}$ \\ ${ }^{1}$ School of Materials and Metallurgy, Northeastern University, Shenyang, 110819, P.R.China \\ ${ }^{2}$ School of Mechanical, Materials and Mechatronic Engineering, University of Wollongong, NSW \\ 2522, Australia \\ azhangjun357@126.com, bhding@263.net, cjwzhaocn@gmail.com
}

Keywords: Pre-quenching process, Q-P process, Microstructure, Mechanical properties, Work hardening.

\begin{abstract}
A refined microstructure consisting of martensite and retained austenite at room temperature has been produced in a Nb-microalloyed low carbon $\mathrm{Si}-\mathrm{Mn}$ steel by a novel heat-treatment, pre-quenching prior to quenching and partitioning processes (Q\&Q-P). The results showed that, compared with the conventional quenching and partitioning steel, the mechanical properties of steel obtained by the novel treatment have been significantly improved, with a good combination of ultimate tensile strength (1000MPa) and total elongation (above 30\%). Meanwhile, the volume fraction of retained austenite has been increased. It was found that the improvement of mechanical properties was mainly attributed to the enhanced TRIP effect due to the relatively high fraction of metastable retained austenite at room temperature. The increased stability of austenite results from the $\mathrm{C}$ and $\mathrm{Mn}$ partitioning during intercritical annealing, which increased the chemical stability of austenite. The formation of refined austenite at intercritical annealing also had a positive effect on the stability of the austenite. As a consequence, the volume fraction of retained austenite at room temperature was significantly increased. Compared with the Q-P steel, the Q\&Q-P steel exhibited higher work hardening exponents during the stage of TRIP effect and had the higher ductility.
\end{abstract}

\section{Introduction}

During the past decades, the automobile industry has shifted its focus towards to the weight reduction and sufficient passenger safety. The steel industry has faced this challenge by offering high strength steels with multiphase, such as bainite, martensite and retained austenite [1-5]. These steels have been designated as advanced high strength steels (AHSS). Among AHSS, low alloyed quenching and partitioning (Q-P) steels have received extensive attention as they offer an excellent combination of high strength and ductility at affordable costs [6-8]. The Q-P process usually consisted of a two-step heat treatment. First, the sheets are held at a temperature in the full austenite $(\gamma)$ or intercritical $(\gamma+\alpha)$ range, and then quenched to a temperature between the martensite-start $\left(M_{s}\right)$ and martensite-finish $\left(M_{f}\right)$ temperatures. Second, the quenched sheets are held either at, or above, the initial quenching temperature in order to enrich the untransformed austenite by carbon diffusion from supersaturated martensite [11,12]. Q-P steels for cold sheets were developed by using manganese and silicon as the main alloying elements in order to suppress the cementite precipitation during the partitioning process $[9,10]$. In this case, a significant amount of austenite 
can be retained after final quenching to room temperature. Compared with TRIP steels, Q-P steels exhibit higher strength, but lower ductility. The lower ductility of Q-P steels is mainly due to lower volume fraction of retained austenite (RA) which is associated with TRIP effect [13]. If the volume fraction of retained austenite increases without significant decrease in strength, the mechanical properties can be further improved. Thus, it is relevant to develop novel methods to increase the volume fraction of retained austenite in Q-P steels.

A recent study indicated that cyclic intercritical tempering heat treatment was beneficial to getting both fine microstructure and stable retained austenite due to the refinement of austenite grains [14]. A reduction in the austenite grain size is well known to increase the austenite stability by suppressing the martensite transformation. The strengthening of austenite by grain size reduction (Hall-Petch effect) increases the mechanical free energy required to transform to martensite. The kinetics of the martensite transformation is also efficiently retarded in the presence of a sufficiently high dislocation density in austenite [15]. Subsequently, Cao et al [16] proposed an ART (austenite reversed transformation) heat treatment which contained an annealing at intercritical annealing temperature for long time after accelerated cooling. The martensite was gradually transformed into a superfine ferrite and austenite duplex structure by austenite reverted transformation (ART) during the annealing process. This annealing heat treatment results in a large volume fraction of retained austenite $(34 \%)$ and an excellent combination of strength $(\sim 1000 \mathrm{MPa})$ and total elongation $(>40 \%)$ at room temperature for middle manganese steels [16]. In consideration of the above mechanisms, the pre-quenching process (from full-austenization temperature to room temperature) had been performed before the quenching and partitioning treatment in order to obtain both fine microstructures and metastable retained austenite. This pre-quenching treatment was employed in the study on a TRIP steel [17], but the performance on Q-P steels was never reported. Hence, the purpose of the present study is to investigate the effect of the pre-quenching process on the microstructural evolution and mechanical properties of the experimental Q-P steel. Also, the effect on the volume fraction of retained austenite and work hardening behaviors is studied.

\section{Experiment}

The chemical compositions of the experimental steel are presented in Table 1. The identified compositions are mainly based on the role of alloying elements, simultaneously considering cost savings of production, which has been discussed in our previous work [18]. A $40 \mathrm{~kg}$ casting ingot was manufactured by a vacuum induction melting method. The ingots were homogenized at $1200{ }^{\circ} \mathrm{C}$ for $2 \mathrm{~h}$, and then hot forged to slabs with width of $100 \mathrm{~mm}$ and thickness of $30 \mathrm{~mm}$. These bars were soaked at $1200{ }^{\circ} \mathrm{C}$ for $2 \mathrm{~h}$ and then hot rolled to sheets with thickness of $4 \mathrm{~mm}$ after eight passes rolling with the starting and finishing temperatures of $1150{ }^{\circ} \mathrm{C}$ and $850{ }^{\circ} \mathrm{C}$, respectively. Subsequently, these sheets were air cooled to room temperature and then cold rolled to strips with thickness of $1.2 \mathrm{~mm}$ after pickling in $10 \%$ hydrochloric acid.

Table 1 Chemical compositions (wt.\%) and critical temperatures $\left({ }^{\circ} \mathrm{C}\right)$ of the experimental steel

\begin{tabular}{|c|c|c|c|c|c|c|c|}
\hline $\mathrm{C}$ & $\mathrm{Si}$ & $\mathrm{Mn}$ & $\mathrm{Al}$ & $\mathrm{Nb}$ & $A_{c 3}\left({ }^{\circ} \mathrm{C}\right)$ & $A_{c l}\left({ }^{\circ} \mathrm{C}\right)$ & $M_{s}\left({ }^{\circ} \mathrm{C}\right)$ \\
\hline 0.18 & 1.48 & 1.44 & 0.15 & 0.025 & 890 & 735 & 390 \\
\hline
\end{tabular}

In order to design an appropriate heat treatment process, the critical temperatures of $A c_{1}, A c_{3}$ and $M_{s}$ of the experimental steel were first obtained by dilatometer tests and were also listed in Table 1. The specimens were divided into two groups. The first group (named Q-P) was austenitized at $910{ }^{\circ} \mathrm{C}$ for $3 \mathrm{~min}$, and then quenched to $220{ }^{\circ} \mathrm{C}$. Subsequently, these quenched specimens were 
held at $400{ }^{\circ} \mathrm{C}$ in a time range from $5 \mathrm{~s}$ to 500s. Finally, a second quenching process was carried out to room temperature. The second group (named Q\&Q-P) was treated by a pre-quenching process, from $910{ }^{\circ} \mathrm{C}$ to room temperature, preferentially to the whole heat treatment. Then the samples were austenitized at $850{ }^{\circ} \mathrm{C}$ for $3 \mathrm{~min}$ followed by quenched to $220{ }^{\circ} \mathrm{C}$ and partitioned at $400{ }^{\circ} \mathrm{C}$ from 5 s to 500 s. Finally, the partitioned samples were quenched to room temperature. The experiments with different pre-quenching process, such as different austenitizing temperatures and time, had been done. It was found that the original austenite grain size of the experimental steel after pre-quenching process increases with the austenitizing temperature and time, and, the original microstructure (prior to quenching and partitioning) under this condition $\left(910^{\circ} \mathrm{C}, 3 \mathrm{~min}\right)$ was the smallest. The purpose of the pre-quenching treatment was to get the microstructure with fine lath martensite, which further refined the austenite grains during the subsequent austenitizing process. Thus, the above heat treatment had been adopted in this work. The schedule for heat-treatment cycle is schematically shown in Fig. 1.
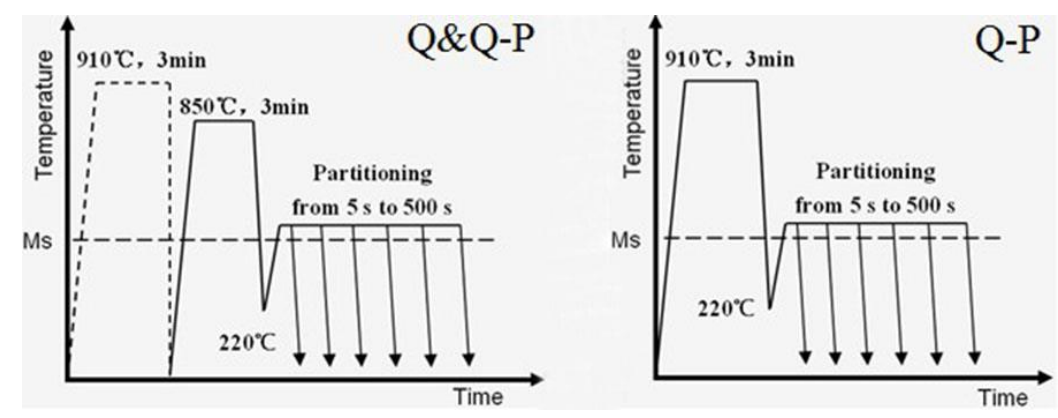

Fig. 1 Schematic heat cycle used in this study

Rectangular tensile specimens (width $12.5 \mathrm{~mm}$, thickness $1.2 \mathrm{~mm}$, and gauge length $25 \mathrm{~mm}$ ) were prepared along the rolling direction after heat treatment. Tensile tests were performed on a SANSCMT-5000 tensile machine at room temperature with a strain rate of $2 \times 10^{-3} \mathrm{~s}^{-1}$. Microstructures were analyzed by scanning electron microscope (SEM). The volume fraction of RA was measured via X-ray diffraction (XRD) with $\mathrm{Cu} \mathrm{K \alpha}$ radiation operated at $40 \mathrm{kV}$ and $100 \mathrm{~mA}$. Samples were scanned from $40^{\circ}$ to $120^{\circ}$ at a scanning rate of $2^{\circ} / \mathrm{min}$. The volume fraction of $\mathrm{RA}\left(V_{\gamma}\right)$ was calculated by using the following Eq. 1 [19,20]:

$$
V_{\gamma}=\frac{1.4 I_{\gamma}}{I_{\alpha}+1.4 I_{\gamma}}
$$

where $I_{\gamma}$ and $I_{\alpha}$ are the integrated intensities of austenite and ferrite diffraction lines, repectively.

In the present work, the diffraction lines of $(200)_{\alpha},(211)_{\alpha},(200)_{\gamma},(220)_{\gamma}$ and $(311)_{\gamma}$ were employed to determine the value of $V_{\gamma}[20]$.

The work hardening behaviors were analyzed by using the following Hollomon equation: [21]

$$
\sigma_{t}=K \varepsilon_{t}^{n_{i}}
$$

where $\sigma_{t}$ and $\varepsilon_{t}$ are the true stress and true strain, respectively, $K$ is the strength coefficient, and $n_{i}$ is the instantaneous work hardening exponent.

The instantaneous work hardening exponent, $n_{i}$, can be deduced from the Eq. 2

$$
n_{i}=\left(\varepsilon_{t} / \sigma_{t}\right)\left(d \sigma_{t} / d \varepsilon_{t}\right)
$$

where the $n_{i}, \sigma_{t}$ and $\varepsilon_{t}$ in Eq. 3 are the instantaneous work hardening exponent, the true stress and the 
true strain, respectively.

\section{Results and discussion}

Fig. 2a and $\mathrm{b}$ show the typical SEM micrographs of samples treated by Q-P and Q\&Q-P heat-treatments, respectively. It is clear that the microstructure of Q-P sample (Fig. 2a) is almost composed of full martensite and this microstructural characterization after Q-P treatment has also been reported elsewhere [22]. By contrast, the microstructural constituents of Q\&Q-P sample consists of ferrite and martensite. The ferrite appears as a lath shape which distributes uniformly in the matrix, whereas the martensite appears as lath beams and distributes in cluster. Meanwhile, the samples treated by Q\&Q-P process exhibit a fine microstructure. The fine microstructure is due to the pre-quenching step that produced fine martensite, which leads to increase density of nucleation sites for austenite during intercritical annealing, resulting in fine austenite. Previous research on TRIP steels pointed out that fine microstructure was beneficial for stabilizing retained austenite and maintaining a relatively high rate of work hardening at larger strain because of the TRIP effect [22]. It should be mentioned that the retained austenite is hard to be distinguished in the SEM micrographs for both samples.
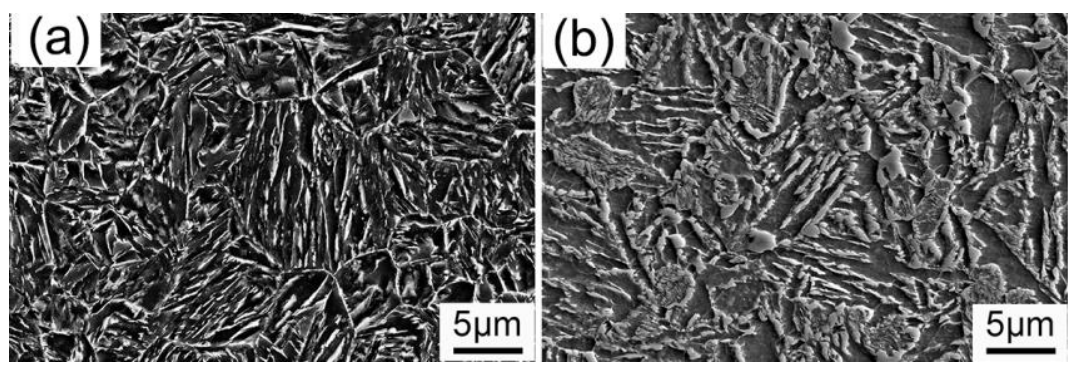

Fig. 2 Typical SEM micrographs of the samples treated by Q-P and Q\&Q-P processes:

(a) after Q-P process, (b) after Q\&Q-P process.
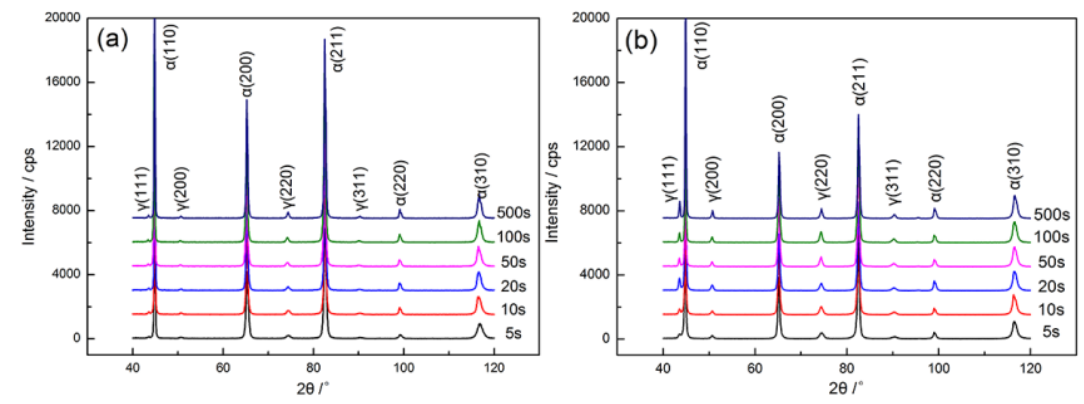

Fig. 3 XRD patterns of Q-P and Q\&Q-P samples and the calculated volume fraction of retained austenite: (a) Q-P samples, (b) Q\&Q-P samples

Table 2 Measured volume fraction (\%) of retained austenite in Q-P and Q\&Q-P samples

\begin{tabular}{|c|c|c|c|c|c|c|}
\hline & $5 \mathrm{~s}$ & $10 \mathrm{~s}$ & $20 \mathrm{~s}$ & $50 \mathrm{~s}$ & $100 \mathrm{~s}$ & $500 \mathrm{~s}$ \\
\hline Q-P & 13.9 & 11.6 & 11.3 & 10.5 & 12.1 & 11.2 \\
\hline Q\&Q-P & 19.3 & 19.9 & 22.5 & 20.4 & 20.4 & 21.6 \\
\hline
\end{tabular}

Fig. 3 shows the XRD patterns of Q-P and Q\&Q-P samples and the calculated volume fraction of retained austenite is illustrated in Table 2. It is clear that the intensity of diffraction peaks of austenite in the Q\&Q-P samples is greater than that in the Q-P samples. Compared with the Q-P treatment, the volume fraction of retained austenite has been significantly increased by Q\&Q-P treatment. As mentioned above, the pre-quenching process was beneficial to getting the 
microstructure with fine martensite, which led to the formation of fine austenite grains during annealing at $850{ }^{\circ} \mathrm{C}$. Smaller grains of austenite was found to be more stable during the quenching due to a resultant lowering of the martensite starting temperature [23,24], which resulted in higher volume fraction of retained austenite at room temperature. Additionally, the Q\&Q-P process adopted intercritical annealing at $850{ }^{\circ} \mathrm{C}$. The enrichment of $\mathrm{C}$ and $\mathrm{Mn}$ in austenite during the intercritical annealing had a positive impact on stabilizing austenite, which led to a relatively lower martensitic starting temperature and decreased the volume fraction of austenite transformed during the consequent quenching. These are currently the underlying reasons for Q\&Q-P samples to have the higher retained austenite fraction than the Q-P samples. An in-depth study is in progress.
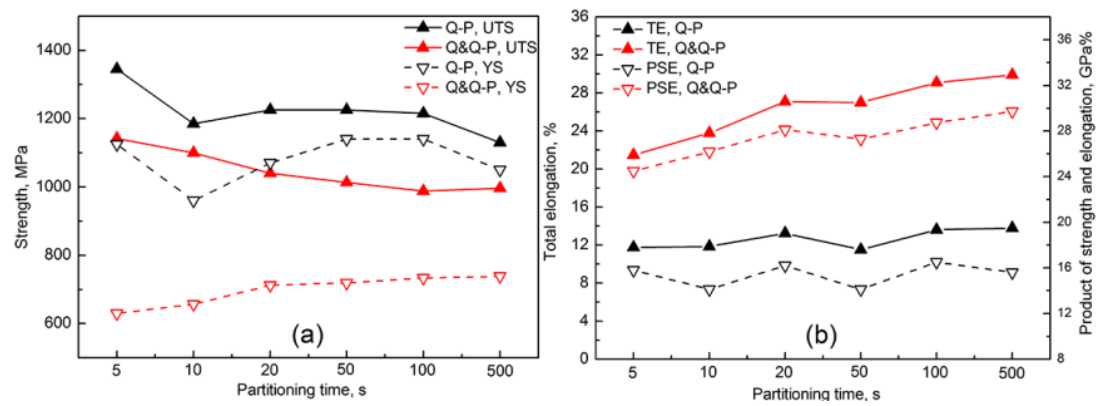

Fig. 4 Mechanical properties of Q-P and Q\&Q-P samples after different partitioning time: (a) ultimate tensile strength (UTS) and yield strength (YS), (b) total elongation (TE) and product of strength and elongation (PSE)

Fig. 4 shows the mechanical properties of the Q-P and Q\&Q-P samples. It can be seen that, compared with the Q\&Q-P samples, the Q-P samples exhibit relatively high ultimate tensile strength but lower total elongation. The YS, UTS and TE of Q-P sample with best PSE are 1140 $\mathrm{MPa}, 1215 \mathrm{MPa}$ and $13.62 \%$, respectively. While the values of Q\&Q-P sample with best PSE are $738 \mathrm{MPa}, 996 \mathrm{MPa}$ and 30.5\%, respectively. The high strength of Q-P samples is attributed to its full martensite structure which results from quenching after austenitizing. By contrast, the additional pre-quenching and the adoption of intercritical annealing are proposed in Q\&Q-P samples. As a consequence, as shown in Fig. 2b, the microstructural constituents consist of ferrite and martensite. Ferrite presented in the experimental steel has a positive effect of improving the plasticity. Due to its low strength and good ductility, ferrite deforms preferentially during deformation. It delays the plastic deformation of martensite and the TRIP effect of retained austenite, which enhances the plasticity of the experimental steel. It can be seen from Fig. 4, although the Q\&Q-P samples exhibit a decrease in strength, the total elongation of Q\&Q-P samples is obviously improved. Compared with the Q-P samples, the PSE of the Q\&Q-P samples is significantly enhanced and reaches $30.4 \mathrm{GPa} \%$, while the value for the Q-P sample is $16.4 \mathrm{GPa} \%$.

The plots of stress-strain and $n_{i}-\varepsilon_{t}$ of the Q-P and Q\&Q-P samples with different partitioning time were illustrated in Fig. 5. It can be seen that, both the Q-P and Q\&Q-P samples exhibit a two-stage strain hardening behavior. In the first stage, the instantaneous strain hardening exponent $n_{i}$ for all the samples significantly decreases with strain. In the second stage, the decrease rate of $n_{i}$ declines and begin to flatten. The differences are, compared with the Q-P samples, the Q\&Q-P samples has more smooth transition from stage-I to stage-II and more durable work hardening behavior. Due to the microstructure of Q-P samples, consisting of martensite and retained austenite, martensite deforms plastically in the early strain stage. As martensite possesses high strength and low formability, the local stress concentration occurs when the strain is small, and then, retained austenite will transform in order to relieve the local stress concentration and is exhausted at small strain stage. For the Q\&Q-P samples, however, the ferrite deforms preferentially in early stage due 
to its low strength and good ductility. The deformation of ferrite results in work hardening which leads to a gentle decline of strain hardening exponent. The second stage of work hardening can be explained by the TRIP effect of retained austenite. Previous studies had shown that the instantaneous work hardening exponents of TRIP steels exhibited increasing and reached a large plateau before break during the TRIP effect stage [25]. Based on the study by Chiang et al. [17], the observed difference in work hardening behavior can be explained in terms of different volume fraction of retained austenite in the Q-P and Q\&Q-P samples. The volume fraction of retained austenite in the Q-P samples is lower than that in the Q\&Q-P samples. Because of the higher volume fraction of retained austenite, the Q\&Q-P samples still have some retained austenite which could transformed at high strains, whereas the Q-P samples have exhausted its retained austenite because of the TRIP effect. Thus, the Q\&Q-P samples exhibit a higher $n_{i}$ value and a larger plateau of work hardening than the Q-P sample at the second stage.

As is shown in Fig. $5 b$ and d, there is a shake in the stress-strain curves and work hardening exponents of the Q\&Q-P sample partitioning for 500s. The shake is thought to be a discontinuous yielding caused by the generation of Cottrell atmosphere. The previous work of Zhao et al. [26] indicated that the yield strength of ultra-low carbon steel increased with the increase of aging time, and the strengthening was caused by the interaction of dislocations and the Cottrell atmosphere. Carbon has strong diffusion ability when partitioned at $400{ }^{\circ} \mathrm{C}$, and it could diffuse sufficiently and concentrates around dislocations to generate the Cottrell atmosphere which has the effect of solution strengthening. Due to the ferrite has low strength and preferentially deforms during deformation, the above strengthening mechanism is obvious in the Q\&Q-P samples. This is the reason why the yield strength of Q\&Q-P samples increases with the increase of partitioning time. However, for the Q-P samples, consisting of retained austenite and martensite, martensite has high strength itself due to the high density of defects (dislocation, lattice distortion and lath boundaries). With the increase of partitioning time, the strongly recovery of martensite results in the decrease of defects in martensite, which leads to the significant decrease in strength of martensite. The interaction of martensite recovery and the strengthening mechanism mentioned above makes the yield strength of Q-P samples presents different changing trends compared with the Q\&Q-P samples. The effect of this phenomenon on the Q-P steels will be analyzed in future work.
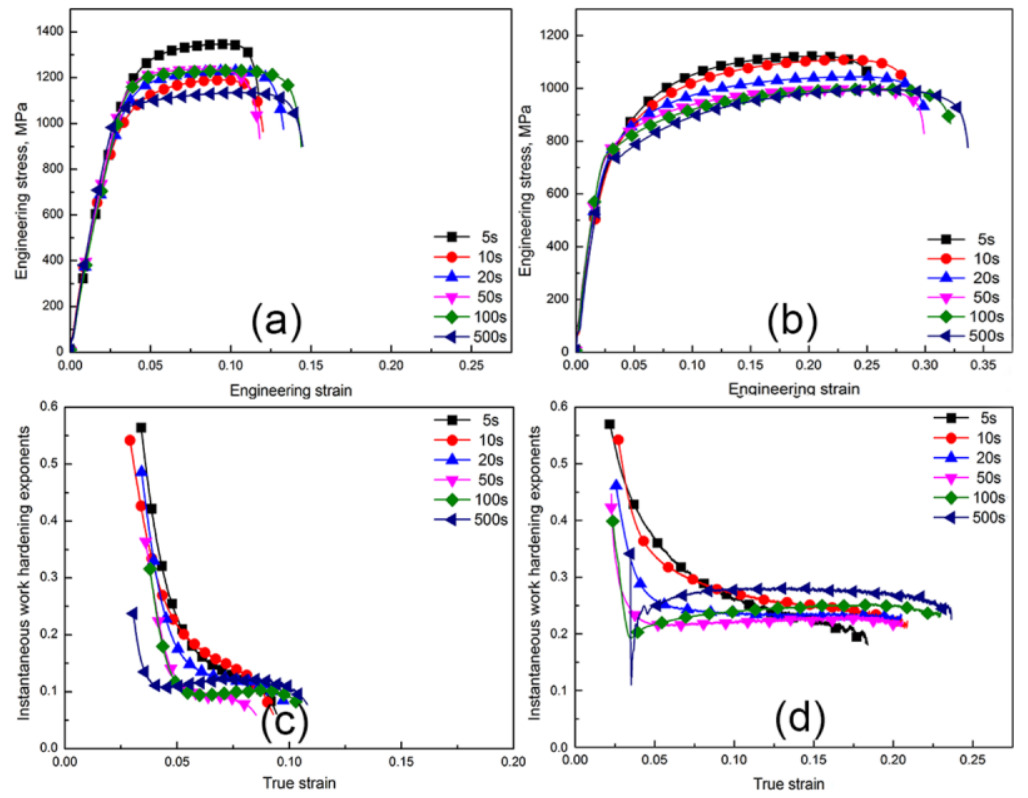

Fig. 5 Stress-strain curves (a, b)and variation instantaneous work hardening exponents (c, d) with true strain for Q-P and Q\&Q-P samples after different partitioning time: (a) (c) Q-P samples, (b) (d) Q\&Q-P samples 


\section{Conclusion}

In this investigation, the pre-quenching process was proceeded prior to quenching and partitioning in a low carbon microalloyed steel, which was conceived to obtain superior mechanical properties. The microstructural features and mechanical properties were studied in details by SEM, XRD and tensile test. The main conclusions were summarized as follows:

1. In the SEM micrographs, the constituent of the Q-P sample are almost composed of lath martensite, while the microstructural constituents of the Q\&Q-P sample are ferrite and martensite.

2. Compared with Q-P treatment, the Q\&Q-P treatment is beneficial to significantly improve the total elongation. Also, the PSE of the Q\&Q-P sample is much higher than that of the Q-P sample.

3. The Q\&Q-P treatment is beneficial to getting stable RA and increasing the volume fraction of RA. As a result, the Q\&Q-P samples could maintain relatively higher exponents of work hardening than the Q-P ones at the large strain stage, which is mainly due to the sustained TRIP effect caused by the higher volume fraction of RA in them.

\section{Ackonwledgments}

The present study was financially supported by the National Natural Science Foundation of China (No: 51031001).

\section{References}

[1] R. G. Davies, Metall. Trans. A 9 (1978) 451.

[2] I. Tsukatani, S. Hashimoto, T. Inoue, ISIJ Int. 31 (1991) 992.

[3] Y. Sakuma, O. Matsumara, H. Takechi, Metall. Trans. A 22 (1991) 489.

[4] Y. Sakuma, D. K. Matlock, G. Krauss, Metall. Trans. A 23 (1992) 1221.

[5] J. A. Jimenez, M. Carsi, O. A. Ruano, et al., Mater. Sci. Eng. A 508 (2009) 195.

[6] S. S. Nayak, R. Anumolu, R. D. K. Misra, et al., Mater. Sci. Eng. A 498 (2008) 442-456.

[7] J. G. Speer, D. K. Matlock, B. C. Cooman, et al., Acta Mater. 51 (2003) 2611.

[8] D. K. Matlock, V. E. Brautigam, J. G. Speer, Mater. Sci. Forum 1089 (2003) 426-432.

[9] A. J. Clarke, J. G. Speer, D. K. Matlock, et al., Scripta Mater. 61 (2009) 149-152.

[10] A. J. Clarke, J. G. Speer, M. K. Miller, et al., Acta Mater. 56 (2008) 16-22.

[11] I. Tamura, Met. Sci. 16 (1982) 245.

[12] O. Matsumura, Y. Sakuma, Y. Ishii, et al., ISIJ Int. 32 (1992) 1014.

[13] O. Matsumura, Y. Sakuma, H. Takechi, Trans. ISIJ 27 (1987) 570-579.

[14] J. W. Morris, Z. Guo, C. R. Krenn, et al., ISIJ Int. 41 (2001) 599-611.

[15] J. Mahieu, J. Maki, B. C. De Cooman, et al., Metall. Mater. Trans. A 33A (2002) 2573-2580.

[16] W. Q. Cao, C. Wang, J. Shi, et al. Mater. Sci. Eng. A 528 (2011) 6661-6666.

[17] J. Chiang, B. Lawrence, J. D. Boyd, et al., Mater Sci Eng A 528 (2011) 4516-4521.

[18] J. Zhang, H. Ding, C. Wang, et al., Mater Sci Eng A 585 (2013) 132-138.

[19] Z. C. Wang, S. J. Kim, C. G. Lee, et al., J Mater Process Technol 151 (2004) 141-145.

[20] Z. Li, D. Wu, ISIJ Int 46 (2006) 121-128.

[21] J. H. Hollomon, Trans AIME 162 (1945) 268-290.

[22] K. Zhang, M. H. Zhang, Z. H. Guo, et al., Mater. Sci. Eng. A 528 (2011) 8486-8491.

[23] W. C. Leslie, R. L. Miller, ASM Trans. Q 57 (1964) 972-979.

[24] D. Q. Bai, A. D. Chiro, S. Yue, Mater. Sci. Forum 284-286 (1998) 253-260.

[25] R.J. Moat, S.Y. Zhang, J. Kelleher, et al., Acta Mater. 60 (2012) 6931-6939.

[26] J. Z. Zhao, A. K. De, B. C. De Cooman, Mater. Lett. 44 (2000) 374-378. 